\title{
Acknowledgment
}

The editors wish to thank the South Asia Regional Studies Center at the University of Pennsylvania for funds to assist in the preparation of the manuscript. 



\section{The Age of Partnership}


